\title{
Estimating Frequencies of Emotions and Actions: A Web-based Diary Study
}

\author{
NORMAN R. BROWN ${ }^{1 *}$, REBECCA L. WILLIAMS ${ }^{1}$, \\ ERIN T. BARKER ${ }^{2}$ and NANCY L. GALAMBOS ${ }^{1}$ \\ ${ }^{1}$ University of Alberta, Canada \\ ${ }^{2}$ National Institute of Child Health and Human Development, Maryland, USA
}

\begin{abstract}
SUMMARY
Mental health questionnaires often ask respondents to report how frequently they experience different emotions. We report two experiments designed to assess the accuracy of these reports and the strategies used to generate them. Each day for 2 weeks, participants in Experiment 1 filled out a webbased emotions-and-activities checklist. Then, they estimated the diary-period frequency of these emotions and activities and indicated how they generated each estimate. In Experiment 2, participants provided frequency estimates and strategy reports, but did not fill out the checklist. We found that (a) the frequency estimates were quite accurate for emotions and activities, (b) participants relied on memory-based strategies (enumeration and direct retrieval) when estimating activity frequencies, but (c) used self-knowledge strategies (personality beliefs and schematic inferences) somewhat more than memory strategies for emotions and (d) the relationship between strategy use and question type was unaffected by diary keeping. We conclude by considering practical and theoretical implications. Copyright (C) 2007 John Wiley \& Sons, Ltd.
\end{abstract}

Health surveys, mental health questionnaires and clinical interviews often include emotion frequency questions. These questions require respondents to indicate the number of times they have experienced a particular emotion (e.g. sadness, anger and anxiety) over a specified period of time (e.g. 1 week, 2 weeks, 30 days, past year; American Psychiatric Association, 1994; Center for Epidemiological Studies Depression Scale [CES-D], Radloff, 1977; Composite International Diagnostic Interview [CIDI], World Health Organization, 1990). Experiment 1 was conducted to address two issues raised by this common practice. First, we wanted to determine whether people can answer emotion frequency questions accurately; second, we wanted to understand how they answer these questions. To this end, participants completed a web-based emotions-and-activities checklist daily for 14 days and were then asked to estimate the number of days on which they experienced the specific emotions and activities mentioned in the checklist and to indicate how they generated each estimate. This design allowed us to assess the accuracy of behavioural and emotional frequency judgements, to identify the strategies that people used to generate these judgements and to determine whether item type (emotions vs. activities) and strategy selection are related. In Experiment 2, participants provided 
frequency estimates and strategy reports, but did not fill out the daily checklist. This experiment enabled us to determine whether performance on the frequency task was influenced by the requirements of the checklist task, as it is unclear whether diary completion affects participants' frequency estimates (Bolger, Davis, \& Rafaeli, 2003).

Obviously, the accuracy issue is of practical importance; if emotion reports are inaccurate, there would be little reason to include them on surveys or to elicit them in clinical settings. To date, the few studies that have used diaries to assess accuracy of emotional frequency estimates have not been encouraging. For example, Schimmack (2002) concludes that 'absolute accuracy of frequency judgements of emotions is low' (p. 191; see also Schimmack \& Hartmann, 1997; Thomas \& Diener, 1990). Relevant studies, however, have used relatively long time frames for measuring actual emotion frequencies (e.g. frames ranging from 3 to 6 weeks, Cutler, Larsen, \& Bunce, 1996; Thomas \& Diener, 1990), or they have gathered relatively fine-grained frequency reports (e.g. assessing the number of times per day that a given emotion was actually experienced, Cutler et al., 1996; Schimmack \& Hartmann, 1997), both of which likely reduce the accuracy of frequency estimates. In contrast, the current study used a 2-week time frame that is more representative of the ones used in health surveys and mental health questionnaires and made use of a more global measure of actual emotion - the presence or absence of a particular emotional state during the course of a day. We expected that these procedures might produce relatively accurate performance. This expectation was based on research indicating that estimation accuracy is inversely related to the actual frequency of the target events and to the duration of the reference period (Blair \& Burton, 1987).

The second issue we addressed in this study concerns the processes people use to generate their emotion frequency estimates. We know from work on (concrete) event frequencies that people use multiple strategies to generate frequency estimates and that strategy selection has representational implications. In this study we extended our multiple-strategies approach (Brown, 1995, 1997, 2002) to investigate the way(s) that people estimate emotion frequency in an attempt to select between competing representational claims (see below).

It is obvious that emotions and activities differ in many ways, ways that may well affect how frequency relevant information is represented and hence the strategies used to generate frequency estimates. In general, behavioural frequency questions ask about welldefined activities that are temporally circumscribed and undertaken in a deliberate manner. These activities, along with information about the participants and locations, define the events that embody them and the events themselves often play a role in goal-directed event sequences or form part of well-established routines (Brown, 2005; Brown \& Schopflocher, 1998a,b). There are also activities that are explicitly planned for or planned around (e.g. examinations and parties).

These aspects of event processing appear to underlie the creation, maintenance and revision of different forms of frequency relevant information, which in turn provide the input to different estimation strategies. Specifically, the elaborate encoding of events should create the type of readily retrieved memory traces that are accessed when people use enumeration-based estimation strategies; when an action is performed on a regular basis, knowledge of one's own schedule can be used to support a rate-based estimate (e.g. 'I swim twice a week, so I have exercised four times in the past 2 weeks'); and people may explicitly store numerical information about the number of recent or upcoming events if they require planning (e.g. 'I have to attend 3 parties this weekend. Damn it!!'). When 
available, this type of explicitly encoded numerical information can be retrieved and the retrieved value stated in response to a behavioural frequency question.

Unlike activities, emotions typically do not define events nor do emotions link events to one another (Brown \& Schopflocher, 1998b). In addition, emotions do not necessarily have a clear-cut beginning or end; they are sometimes difficult to label, sometimes mixed and sometimes go unnoticed. Moreover, there is no straightforward relation between emotions and the events that elicit them; some types of event can produce diametrically opposed emotions or no emotion at all (e.g. attending a concert), whereas others reliably produce the same affective reaction. Finally, it should be noted that affect can rapidly fade from memory and often appears to be reconstructed when it is solicited after the fact (Levine, 1997).

Given the transitory nature of emotions and the lability of their mnemonic representations, it seemed likely that people may estimate emotional frequencies in a different way than they estimate behavioural frequencies (Bolger et al., 2003). It could be that people estimate emotional frequencies by relying on various forms of self-knowledge, that is beliefs about one's traits and moods and characteristic response patterns. For example, a student who feels she is having a good semester may infer that she is happy on most days; this inference would support both high-frequency estimates for positively valanced terms and low-frequency estimates for negatively valanced terms. Alternatively, it is also possible to generate emotional frequency estimates in the same way people generate estimates when they are required to estimate the number of times that an item with a particular unstated property (e.g. metallic) appeared on a list (Barsalou \& Ross, 1986; Conrad, Brown, \& Dashen, 2003; Pandelaere \& Hoorens, 2006). People generally use a modified type of enumeration to answer these questions; they retrieve potentially relevant instances and determine whether or not the target property is instantiated in the retrieved instance. By extension, one could imagine a person using this enumerate-and-validate strategy to generate emotional frequency responses and that the retrieval process might on some occasions be guided by beliefs concerning the types of event that are known to elicit the target emotion. Interestingly, the two broad types of estimation strategies described here (i.e. self-knowledge and memory-based) map onto theoretical arguments concerning the representation of self.

Specifically, Klein and Loftus (1993) argued for two models of self-knowledge (or how one mentally represents characteristics that describe the self): a pure exemplar view and an abstract summary view. The pure exemplar model assumes that self-knowledge consists of separate representations of relevant behaviours, which are retrieved from specific autobiographical memories in which the behaviour was enacted. This model is exemplified by someone who describes himself as an angry person by recalling specific instances in which he behaved in an angry manner. In this case, self-description is based on episodic knowledge, or recall of particular experiences that have occurred (Robinson \& Clore, 2002a).

On the other hand, the abstract summary model assumes that self-descriptions are abstracted from relevant behaviours that occurred in the past, but are represented more in summary form than in specific autobiographical memories (Klein \& Loftus, 1993). Thus, self-descriptions are based in a generic self-knowledge that evolved over time, with many specific experiences being lost to memory ('I tend to be an angry person'). This generic type of self-knowledge is based on semantic memory, which is comprised of generalisations or beliefs about the self (Robinson \& Clore, 2002a).

Based on judgement latencies for estimating the frequency of emotions, Robinson and Clore (2002b) reported that people relied primarily on instance retrieval for estimating 
emotion frequencies across short time frames (e.g. 2 weeks or less) but their reliance on self-knowledge increased as the time frame grew longer (see also Schimmack, 2002). While the Robinson and Clore study inferred from judgement latencies that the use of enumeration and self-knowledge strategies depended on the time frame, it did not assess the accuracy of emotion recall.

The current study holds the time frame for recall constant (i.e. 14 days), assesses estimation accuracy, and asks participants to indicate how they generated their estimates. We expected that participants would rely on memory-based strategies (enumeration and direct-retrieval) when responding to activity questions. This is because the activities tracked in this study can produce the type of distinctive and readily retrievable memory traces that enable enumeration. The prediction for the emotion items was not as clear. If the exemplar view is correct, then people should rely on enumeration-based strategies to estimate emotion frequencies. For example, a person responding to the 'happy' item might look back over the past 2 weeks and attempt to count the events that made him or her happy during that time. Tentatively, if the self-knowledge view is correct, then people will make estimates of the frequency with which they experienced a specific emotion, not on specific details or recollections, but on generic self-knowledge. For example, the individual might say 'I am generally a happy person so I felt happy every day'. Finally, as the Robinson and Clore (2002b) study suggests, it could be that emotional information is represented in two ways; as self-knowledge and in event memories. If so, we should find evidence that people use both memory-based strategies and selfknowledge strategies when responding to emotion items.

In summary, we conducted a daily diary study that enabled us to determine the degree to which people can provide accurate estimates of their emotional states and their activities on the day after a 14-day reference period. We also included a strategy menu when participants were asked to provide frequency judgements; this allowed us to determine how the participants generated their frequency estimates and whether there was a relation between strategy selection and item type (i.e. emotion vs. activity). This design enabled us to pose three research questions, examining three important issues: (a) what strategies do individuals use to estimate emotion frequencies? (b) do people use different strategies when estimating emotion frequencies compared to strategies used to estimate activity frequencies? (c) Do strategy differences, if they exist, affect the accuracy of estimates of emotion frequency and those of activity frequency?

\section{EXPERIMENT 1}

\section{Method}

\section{Participants}

Participants were 100 undergraduate students (62 females) enrolled in introductory psychology courses at a large Canadian university. All participants were recruited through the introductory psychology research pool and each received research participation credit for involvement in the study. On average, participants were 21.5 years old.

\section{Procedure}

Research assistants met with participants in small groups to explain the study and to have participants sign consent forms. In order to participate, students were required to have 
access to the Internet in the evenings so that they could submit an online (web-based) checklist at the end of each day. All students at the university receive computing accounts and email addresses, $100 \%$ have access to computer labs on campus and $100 \%$ in dormitory rooms have high-speed access.

Part I of the study asked participants to complete an online (web-based) daily checklist of feelings and activities for 14 consecutive days (Day 1-Day 14). Participants accessed the daily checklist via the study website, stored on the Department of Psychology's secure server. Email reminders to complete the online daily checklist, with a link to the study website, were sent on the start date (Day 1) and partway through the study (Day 7). All participants started on a Thursday evening, and the checklists were available from 6:00 p.m. each evening until noon the following day. They were asked to complete the checklist as late as possible before going to sleep each night.

Part II of the study took place on Day 15. Participants were asked to access the study website, but to complete a different questionnaire on Day 15. The Day 15 questionnaire asked participants to estimate the number of days during the past 2 weeks they had experienced emotions and activities they had reported during Part I of the study (Day 1Day 14). In addition, participants were asked to indicate the estimation strategy used to generate their estimates for each target emotion and each target activity. Four versions of the Day 15 questionnaire were created by first counterbalancing the randomised order of the survey options, followed by counterbalancing the randomised order of the questions themselves. New instructions, with a link to the Day 15 questionnaire, were emailed to participants on Day 15.

\section{Measures}

\section{Daily checklist target emotions}

Participants responded 'yes' or 'no' to the following question: Did you feel [target emotion] today? The seven emotions or feelings were energetic, excited, happy, irritable, nervous, sad and upset. Five emotions were selected from the widely used Positive and Negative Affect Schedule (Watson, Clark, \& Tellegen, 1988) to represent pleasurable (energetic and excited) and aversive (irritable, nervous and upset) mood states. Happiness and sadness were also assessed because they are commonly measured in studies examining cognitive processing of emotions (e.g. Smith \& Ellsworth, 1985; Tiedens \& Linton, 2001). Finally, many of these emotion terms appear in frequently used mental health questionnaires or structured interviews which ask individuals to indicate how often they felt the emotion in the past week (e.g. sadness and happiness in the CES-D; Radloff, 1977), in the last month (e.g. upset and nervous in the Perceived Stress Scale; Cohen, Kamarck, \& Mermelstein, 1983), or over a period of 2 weeks in the past 12 months (e.g. sadness and lacking in energy in the CIDI; World Health Organization, 1990).

\section{Daily checklist target activities}

Participants reported the number of alcoholic drinks consumed, the number of minutes of exercise performed, whether they had socialised with friends ('yes' or 'no'), and whether they had written a quiz or test ('yes' or 'no') each day.

\section{Day 15 estimation questionnaire}

First, participants were asked to think back over the past 14 days (the daily checklist period) and report the number of days they had felt each target emotion or had engaged in 
each target activity. Following each estimate, participants indicated which of the six strategies they used to generate each estimate. The six strategy options were (1) Enumeration: 'I thought back over the past 14 days and tried to remember and count each day I [target activity/emotion]', (2) Direct retrieval: 'I immediately remembered the number of days I [target activity/emotion]', (3) Typical: 'I thought of situations in which I generally [target activity/emotion] and figured out how many of those situations occurred in the past 14 days', (4) Never: 'I never or rarely [target activity/emotion]', (5) On-average: 'I thought about how often I [target activity/emotion] on average, and used that to come up with the number of days I [target activity/emotion] in the last 14 days' and (6) Other: 'I used some other method for coming up with my response'. Half of the participants saw the menu options listed in the same order as they are presented above and the other half saw the options (except for option \#6) in the reverse order.

Data collected in a pilot verbal protocol study provided the source of the menu options. Fifteen introductory psychology students were recruited from the research pool and received course credit for their participation in the pilot study. Participants were asked to think back over the past 14 days and estimate the number of days they felt each of the target emotions or engaged in each of the target activities. Pilot study participants were asked to talk through their thinking process aloud. Responses were audio-taped and examined for recall strategy themes. The six menu options were derived from these responses and previous research (Brown, 1995; Conrad, Brown, \& Cashman, 1998).

\section{RESULTS AND DISCUSSION}

\section{Compliance}

Of the 100 participants, 11 did not take part in the Day 15 estimated frequency test. Fifteen of the remaining 89 were fully compliant, filling out the activities-and-emotions checklist each day during the 2 -week reference period. Fourteen completed 13 checklists. Fifty-eight completed between 4 and 12 checklists, and two participants filled out the checklist just once.

Prior to performing any data analysis, the 89 participants who completed the Day 15 estimated frequency test were divided into two groups: a diary-complete group $(n=29)$ and a diary-incomplete group $(n=60)$. Only those who provided at least 13 diary entries during the 2-week reference period were assigned to the former. This post hoc division makes it possible to examine estimation accuracy for the diary-complete group and provides a convenient internal replication; below we report estimate size and strategy use as a function of item type for both diary-complete and diary-incomplete groups.

\section{Actual frequency, estimated frequency and estimation accuracy}

The checklist data obtained from the participants in the diary-complete group were used to determine the observed number of days (actual frequency) on which each person reported each of the target emotions and performed each of the target activities. For all of the emotions and the activity questions dealing with socialising and quiz taking, this was done by tallying all of the positive responses across days. Participants provided numerical responses to the minutes-exercised question and the number-of-alcoholic-drinks-consumed 
question; in both cases, values greater than 0 were treated as positive responses and then tallied.

When this initial tallying was completed, a simple correction was applied to counts obtained from the 14 participants in the diary-complete group who had missed one checklist. This was done by adding one to a tally when it was greater than seven. To assess estimation accuracy, a signed error was computed for each estimate by subtracting the actual frequency for a given item from its estimated frequency. Thus, a positive signed error indicated an overestimate of the number of days for a given target emotion or activity and a negative signed error indicated an underestimate of the frequency of an item. Absolute error was computed from each signed error by taking its absolute value. A large absolute error indicated that estimated frequencies were quite different than the actual frequencies regardless of sign. A small absolute error indicated that the estimated frequencies and the actual frequencies were quite similar.

For each participant in the diary-complete group, mean actual and estimated frequencies and mean signed and absolute errors were generated separately for the emotion items (averaging across all seven items) and for the activity items (averaging across all four items). Similarly, the Day 15 frequency test data were used to compute two estimated frequency means, one for the emotion items and one for activity items, for each participant in the diary-incomplete group. Finally, two rank-order correlations were computed between estimated and actual frequency, for each person in the diary-complete group. One was computed over the seven emotion items and the other over the four activity items.

Figure 1 presents mean actual frequency and estimated frequency as a function of item type. Three observations are clear from this figure. First, for the diary-complete group, actual frequency was greater for the target emotions than for the target activities, $t(28)=5.10, p<0.01, S E=0.42$ (see Figure 1, Panel A). Second, estimated frequencies closely resembled actual frequencies. Third, this held true regardless of whether the estimates were produced by participants in the diary-complete or the diary-incomplete

\section{Diary-Complete Group}

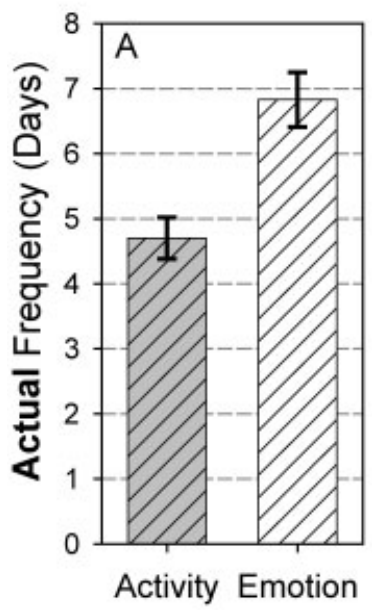

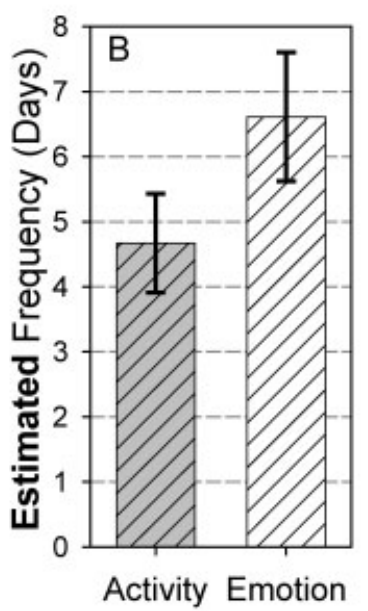

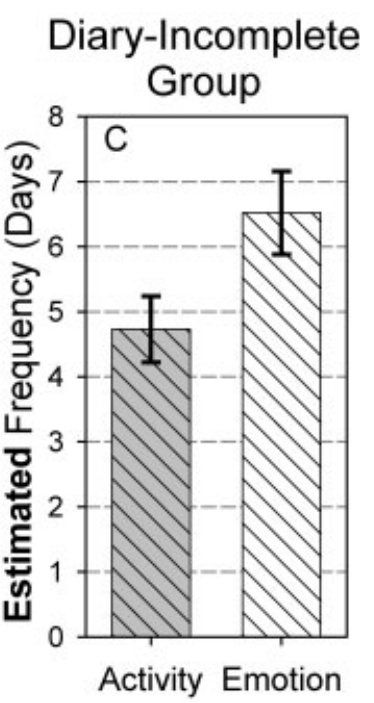

Figure 1. Mean actual frequency for the diary-complete group (Panel A) and estimated frequency for the diary-complete group (Panel B) and the diary-incomplete group (Panel C) as a function of item type. Experiment 1 data 
group. Specifically, a Group (diary-complete vs. diary-incomplete) $\times$ Item Type (emotions vs. activities) repeated measures Analysis of variance (ANOVA), in which item type was a within-subjects factor, indicated that estimated frequency was greater for the target emotions than for the target activities. In this case, the item type difference was reliable, $F(1,87)=32.51, p<0.01, \mathrm{MSE}=4.16$, and the main effect of group and the Group $\times$ Item Type interaction were not $(F<1.0$ in both cases).

The close similarity between the estimated and actual frequencies apparent in Figure 1 means that the estimates were unbiased. Specifically, for the diary-complete participants, mean signed error was -0.22 days for the emotions and -0.03 days for the activities. These values did not differ from 0.0 and were not reliably different from one another $(t<1.0$ in all cases). Not only were the estimates unbiased, they also tended to be quite accurate. Mean absolute error was 0.96 days for activities and 1.96 days for emotions. Although the difference between these means was reliable, $t(28)=5.93, p<0.01, S E=0.17$, it should be noted that error for the emotion estimates was still less than $14 \%$ of the response range. ${ }^{1}$ Moreover, the rank order correlations between estimated and actual frequency were strong for both the emotion items, mean $r=0.68, S E=0.09$, and the activity items, mean $r=$ $0.88, S E=0.06$.

\section{Strategy choice}

During the Day 15 frequency test, strategy reports were collected following each frequency estimate. For each participant, these reports were used to determine a percentage-of-use value for each strategy as a function of item type. Enumeration and Direct-retrieval strategies were classified as memory-based strategies because they drawn on non-generic information in the form of event instances or explicitly encoded, temporally delineated frequency tags. In contrast, Typical and On-average strategies coded as self-knowledge strategies because they rely, not on specific encoded instances or event counts, but on generic beliefs about how often one experiences a particular emotion or activity. The participant's use of any other strategy was considered neither memory- nor self-knowledge based. Figure 2 presents these percentages averaged over all participants (top panel), and averaged separately for participants in the diary-complete (middle panel) and diaryincomplete (bottom panel) groups. It is clear from this figure that: (a) participants were much more likely to use one of the memory-based strategies (enumeration and direct retrieval) than one of the self-knowledge strategies (on-average and typical) when they responded to the activity items, (b) self-knowledge strategies were used somewhat more

\footnotetext{
${ }^{1}$ The data from the diary-complete group indicate that participants sometimes experienced a given emotion or activity every day during the diary period; this was true for $8.4 \%$ of the emotion items and $3.4 \%$ of activity items. Conversely, some participants never experienced a particular emotion or activity: $4.4 \%$ for the emotion terms and $8.6 \%$ for the activity terms. Theoretically, frequency estimates and their accuracy could be affected by averaging these extreme items (items which should elicit very accurate estimates) with items that were checked off intermittently (which may be more difficult to estimate). To examine this possibility, we eliminated the extreme items and then re-computed a mean estimated frequency, a mean signed error and a mean absolute error for the remaining emotion and activity items, for each participant in the diary-complete group. This procedure had a negligible effect on the pattern of results. Again, item type was related to actual frequency (for emotions, $M=6.55$, for activities, $M=4.75 ; t(28)=3.64, S E=0.49, p<0.01$ ), estimated frequency (for emotions, $M=6.34$, for activities, $M=4.73 ; t(28)=2.79, S E=0.58, p<0.01$ ) and absolute error (for emotions, $M=2.05$, for activities, $M=1.08 ; t(28)=5.73, S E=0.17, p<0.001$ ), but not signed error (for emotions, $M=-0.21$, for activities, $M=-0.02 ; t(28)<1.0$ ). Moreover, the magnitude of the signed errors indicates that responses elicited by non-extreme items were unbiased and the magnitude of the absolute errors indicates that these estimates were fairly accurate.
} 

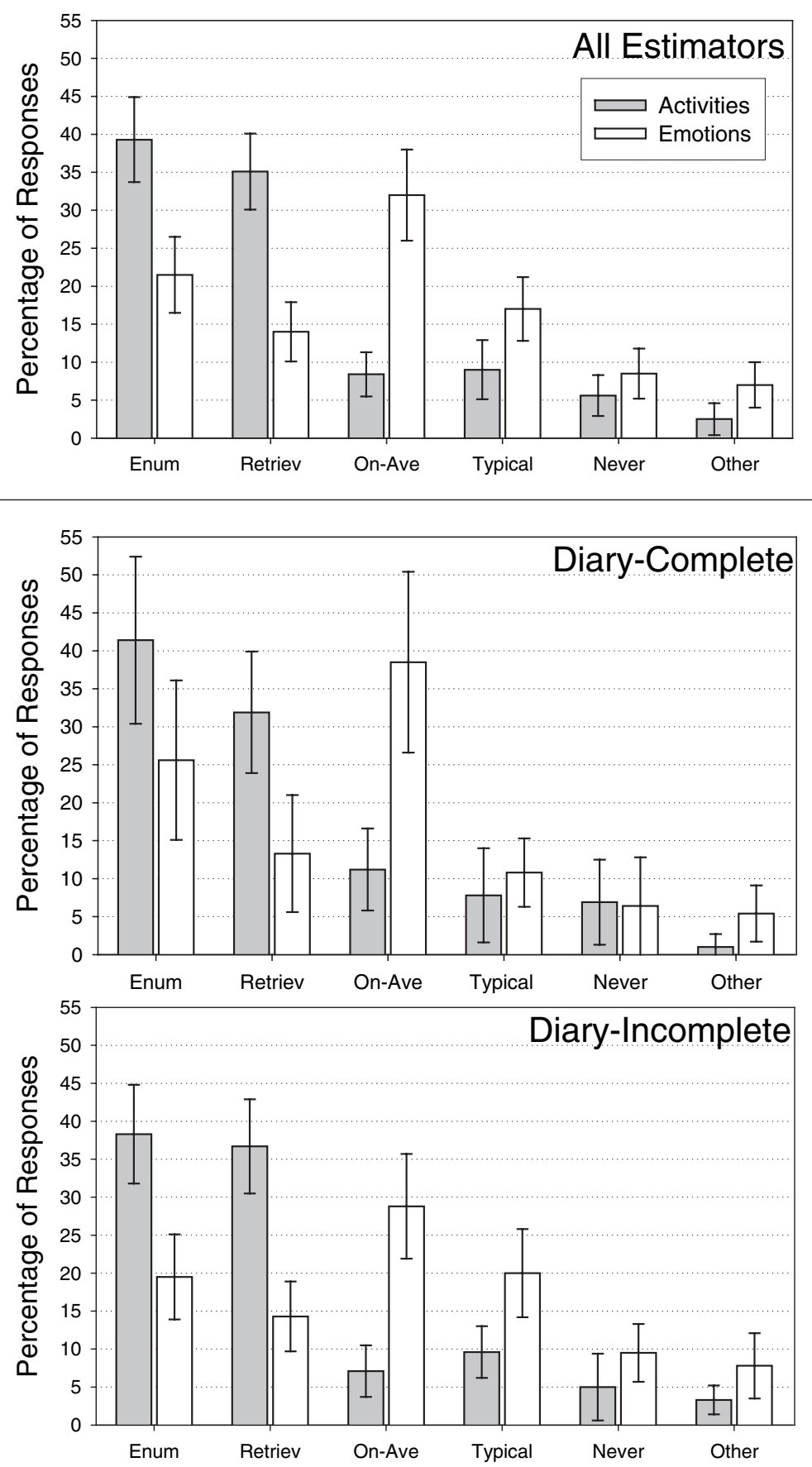

Figure 2. Percentage-of-use for each strategy as a function of item type averaged over all participants (top panel), averaged separately for participants in the diary-complete group (middle panel) and diary-incomplete group (bottom panel). Strategy abbreviations: enum stands for enumeration, retriev stands for direct retrieval, on-ave stands for on-average, typical stands for activities or emotions in typical situations, never indicates that the participants selected the never or rarely option and other indicates that participant indicated using another strategy. Experiment 1 data 
often than memory-based strategies to answer emotion questions, and (c) these tendencies were consistent across groups.

We conducted a Group (diary-complete vs. diary-incomplete) $\times$ Strategy Type (memory-based vs. self-knowledge) $\times$ Item Type (emotions vs. activities) repeated measures ANOVA (with Strategy Type and Item Type within-subjects factors) to confirm these observations. The dependent variable was participant's percentage of use of memorybased strategies (enumeration plus direct retrieval) and self-knowledge strategies (onaverage plus typical) for the activity and emotion items. This analysis produced a reliable Strategy $\times$ Item type interaction, $F(1,87)=97.13, p<0.00001$, MSE $=0.10$ (see Figure 3). The effect of Group was unreliable, and failed to interact with the other factors in the analysis, $F<1.0$. This indicates that willingness or ability to complete the daily dairy had no effect on the relationship between item type and strategy selection

\section{The relationship between strategy choice, item type and actual frequency}

In this experiment, participants were more likely to use memory-based strategies when estimating activity frequencies than when estimating emotion frequencies and they were more likely to use self-knowledge strategies when estimating emotion frequencies than when estimating activity frequencies. We believe that this relation exists because emotions and actions have different mnemonic consequences. There is, however, an alterative explanation. We know that enumeration-based strategies become increasingly less common as frequency increases (Blair \& Burton, 1987; Brown \& Sinclair, 1999; Burton \& Blair, 1991; Conrad et al., 1998). In this experiment, actual frequency was greater for the emotion items than for the activity items. Thus, it could be that this partial confounding of

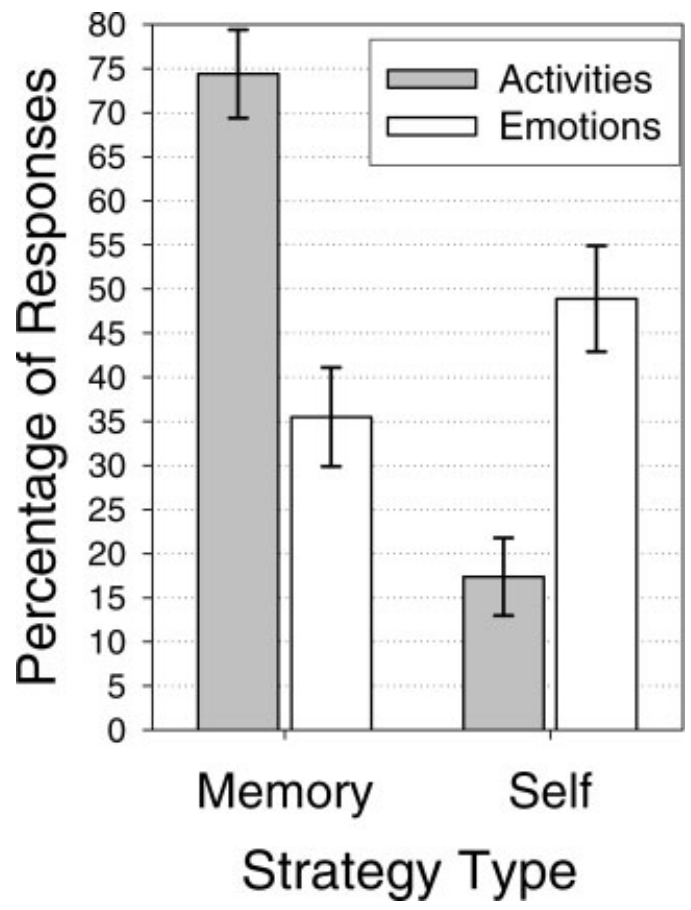

Figure 3. Strategy use as a function of item type. Experiment 1 data 
actual frequency with item type caused the observed relationship between strategy selection and item type.

If this explanation is correct, the ratio of memory-based responses to self-knowledge responses should decrease as actual frequency increases (indicating a relative decrease in the prevalence of enumeration and direct retrieval), and the pattern produced for the emotion items should parallel the pattern produced for the activity items. Two steps were required to determine whether these conditions were met. First, for the diary-complete group, we determined the relationship between actual frequency and strategy selection for both types of items, for low (1-3 days during the diary period), moderate (4-6 days) and high frequency ( 7 or more) items, that is, we computed the percentage of memory-based, self-knowledge based and other strategies out of the total number of strategies reported across all items (separately for activities and emotions) at low, moderate and high frequency levels. These percentages are plotted in Figure 4. Then we computed the necessary ratios for each item type by dividing the percentage of memory-based responses at a given frequency level by the percentage of self-knowledge responses at that same level.

It is clear from this figure that activities and emotions produced very different patterns of strategy use. For the activity items, memory-based strategies dominated performance at low frequencies and the use of these strategies decreased as actual frequency increases. At the same time, self-knowledge strategies were relatively uncommon but unaffected by actual frequency. These trends produced memory-to-self ratios that dropped from 7.11 to 3.74 to 1.11 across the three frequency levels. The corresponding ratios for the emotion items were $0.99,0.57$ and 0.60 . These values indicate that, for the emotion items, selfknowledge responses were at least as common as memory-based responses regardless of frequency.

In brief, the activity items, but not the emotion items, displayed the standard relation between actual frequency and strategy use. The fact that activities and emotions produced

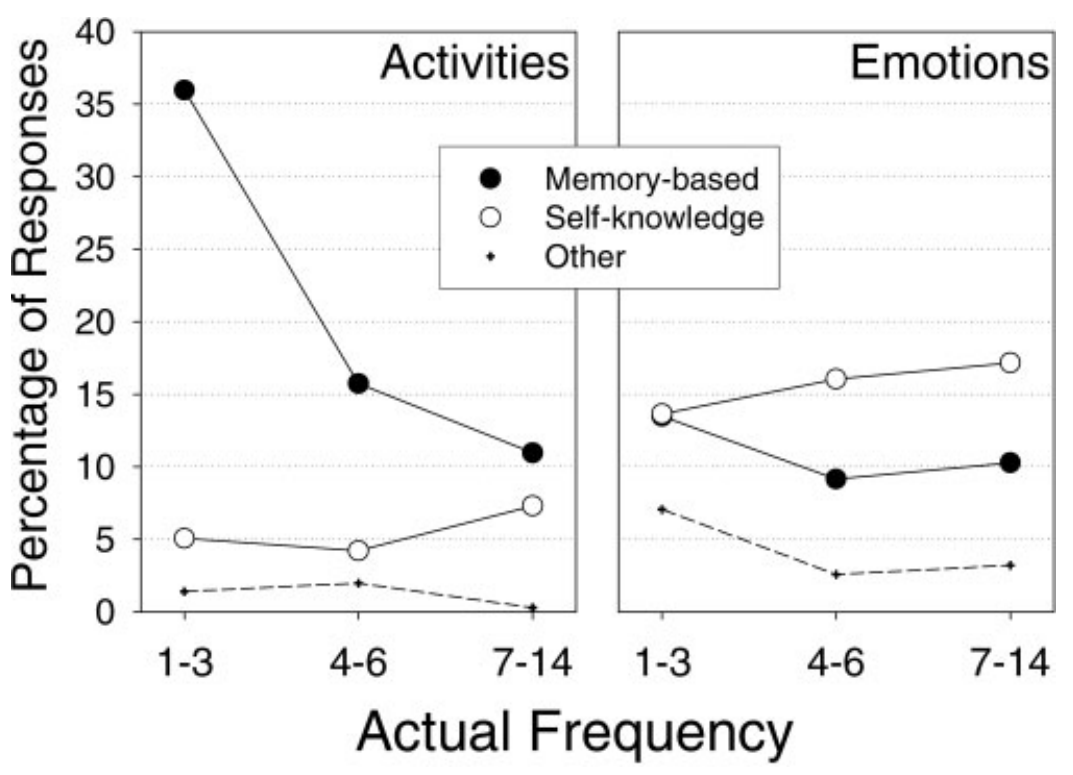

Figure 4. Strategy selection as a function of actual frequency for emotion and action items. Experiment 1 data 
very different patterns of memory-to-self ratios rules out the frequency confound as the explanation for the relation between item type and strategy selection. Instead, the data suggest that the source of this relationship lies in the way that frequency relevant information is represented; it appears that activities are more likely than emotional experiences to produce distinct memory traces that can later be retrieved and counted, and that emotional experiences are more likely to reflect and/or affect the beliefs held about one's self. Of course, specific emotions (i.e. at the item level) can have different frequencies, valences and intensities, all of which may have mnemonic consequences, thereby affecting strategy selection (e.g. Smith \& Ellsworth, 1985; Tiedens \& Linton, 2001). This issue is addressed at the end of Experiment 2, by combining estimated frequency data at the item level for both experiments.

\section{EXPERIMENT 2}

Compliance differences in Experiment 1 made it possible to assign participants to two groups in a principled manner and then to test for possible between-group differences. As noted above, diary-incomplete participants were virtually indistinguishable from diarycomplete ones; participants in both groups produced higher frequency estimates when responding to emotion items than when responding to activity items, and they displayed the same pattern of strategy choice. In both cases, activity items were more likely to elicit memory-based responses than emotion items and emotion items were more likely to elicit self-knowledge responses than activity items. Despite this internal replication, we cannot rule out the possibility that performance on the Day 15 frequency test was influenced by the process of monitoring and recording one's activities and emotions during the reference period. After all, even participants in the diary-incomplete group tended to be quite conscientious; on average, they filled out $8.70(S E=0.36)$ checklists over the 2-week reference period.

Experiment 2 was conducted to address the possibility that diary activities during a reference period affect frequency estimation. Participants who took part in this experiment simply completed the Day 15 frequency test, but were not required to complete the activities-and-emotions checklists. We expected that the results of the current experiment would replicate those reported above. The expectation was based on prior research indicating that diary keeping has little if any effect on autobiographical memory (Thompson, 1982).

From an applied perspective, it seemed important to produce a diary-free replication of our findings. This is because people asked to report the frequency of their emotions and activities on surveys and in clinical settings typically do not monitor their lives during the instrument-defined reference period. Thus, if this work is to be generalised to applied settings, it is necessary to demonstrate that the relations documented in Experiment 1 are robust in the sense that they can be easily replicated and that they are not an artifact of the diary procedure.

\section{Method}

\section{Participants}

Fifty-two people (39 females) participated in Experiment 2. All attended the same university as those in Experiment 1 . They were recruited through the introductory 
psychology pool, and received course credit for their participation. The average age was 20.7 years.

\section{Procedure}

The same web-based Day-15 frequency questionnaire was used in this experiment as in the last. However, in this experiment, participants did not complete daily diaries prior to filling out the questionnaire. As before, all the participants were met in groups to provide their consent and to receive instructions for completing the web-based aspect of the study. On the Thursday of the week in which the initial meeting took place, participants were asked to sign on to the website after 6:00 p.m. to complete the survey. An e-mail was sent to participants to remind them to complete the checklist.

\section{RESULTS AND DISCUSSION}

As in Experiment 1, mean estimated frequency, percentage strategy use and percentage of memory-based and self-knowledge responses were computed over both item types for each participant. And as expected, these data replicated the main features reported above. As before, (a) frequency estimates were higher for emotion items $(M=4.66, S E=0.20)$ than activity items $(M=3.17, S E=0.21 ; t(51)=3.20, p<0.0001)$; (b) strategy-use patterns differed as a function of item type (see Figure 5); and (c) participants were much more likely to use memory-based strategies than self-knowledge strategies when estimating activity frequencies and were somewhat more likely to use self-knowledge strategies than memory-based strategies when estimating emotion frequencies (see Figure 6). These tendencies produced a reliable Strategy Type $\times$ Item interaction, $F(1,51)=$ 42.97, $p<0.0001, \mathrm{MSE}=0.08$. Thus, it appears that the relationship between strategy use and item type is a robust one and that it neither requires diary keeping nor reflects the mnemonic consequences of providing a daily report on one's feelings and actions.

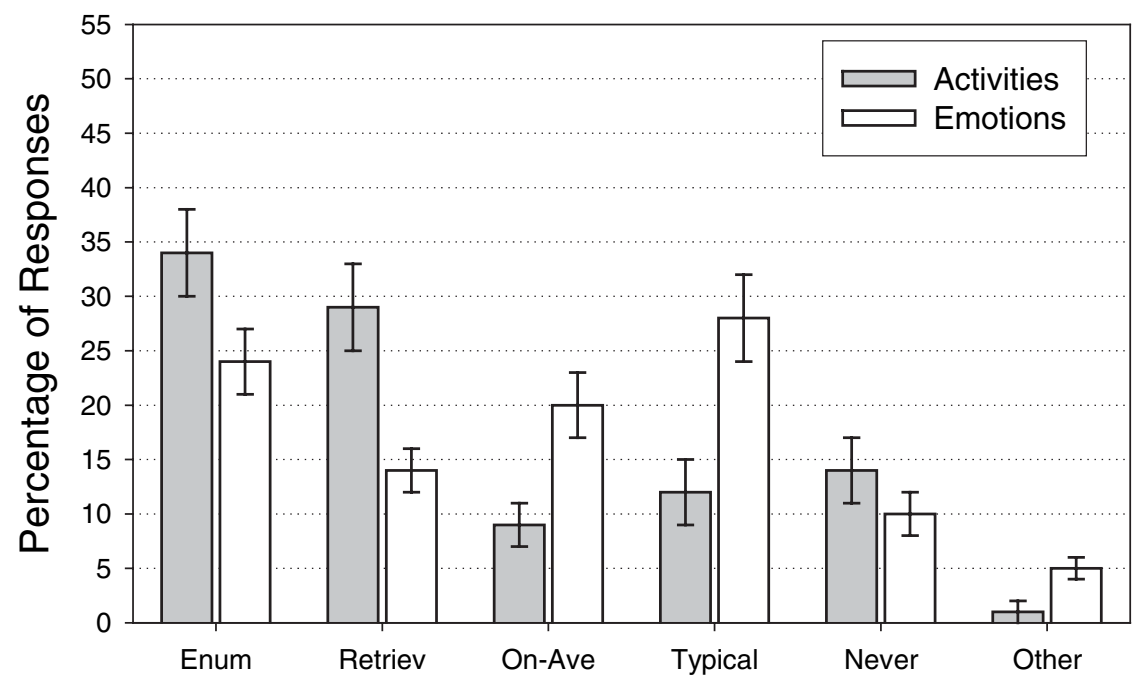

Figure 5. Percentage-of-tise for each strategy as a function of item type. Abbreviations the same as in Figure 2 


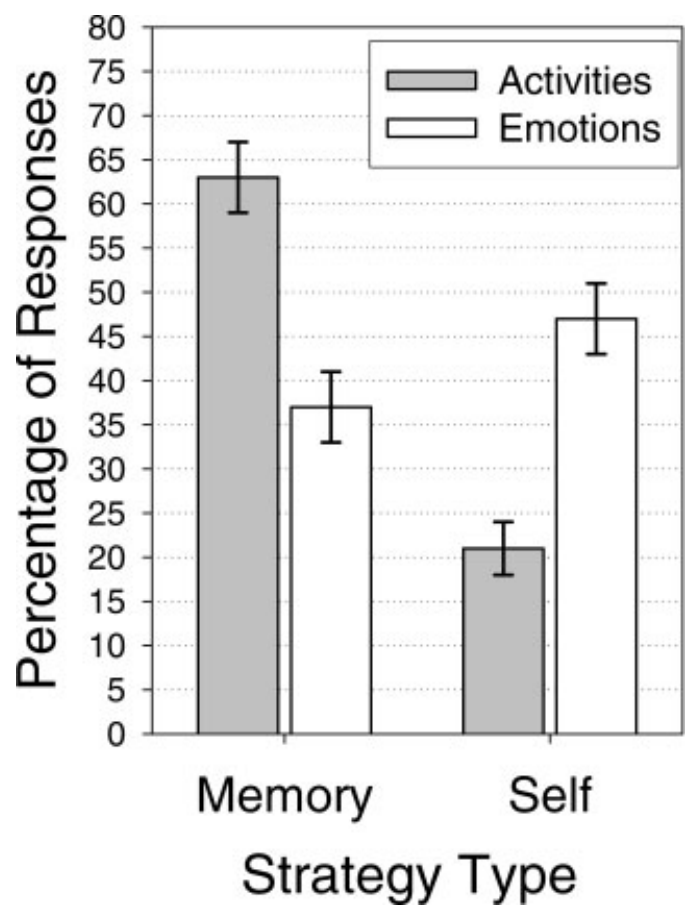

Figure 6. Percentage-of-use for each strategy as a function of item type. Strategy use as a function of item type. Experiment 2 data

To this point in the analyses, emotion items and activity items have been examined globally, by averaging across reports of multiple emotions and activities, respectively. This procedure collapses across items with different actual frequencies, and in the case of emotions, combines those with different valences (i.e. positive and negative). The aggregation of items in this manner may obscure different patterns of strategy selection depending on dimensions like valence. To examine this possibility, estimated frequency and strategy use data from Experiments 1 and 2 were combined and examined at the item level. Table 1 presents the mean estimated frequencies and standard errors separately by item, showing variability in the estimated frequencies across items. The percentages of memory-based, self-knowledge and other strategies used to generate the frequency estimates are also shown for each item. Finally, a chi-square test contrasted the frequency of memory vs. self-knowledge strategies for each item (for these tests, cases were omitted in which other strategies were cited). There is a clear pattern of results, which closely parallel earlier analyses. Regardless of the natural variability that exists across specific items, memory-based strategies were used significantly more often than self-knowledge strategies for all four activities. Despite considerable variability in the estimated frequencies of the emotion items, on six of seven emotions, the percentages for selfknowledge strategies were higher than the percentages for memory-based strategies; these differences reached significance for energetic and irritable. Overall, then there was strong support for the use of memory-based strategies at the item level for activities, and participants were somewhat more likely to use self-knowledge strategies when estimating the frequency of specific emotions. 
Table 1. Mean estimated frequency and standard error, percentage of memory, self-knowledge and other strategies used and chi-square results comparing memory vs. self, by item

\begin{tabular}{lrrrrrc}
\hline Item type and Item & $M$ est. $f$ & $M S E$ & \% Memory & \% Self & \% Other & Chi-Square $(n)$ \\
\hline Emotion & & & & & & \\
$\quad$ Energetic & 6.43 & 0.34 & 28 & 50 & 22 & $8.18(110)^{*}$ \\
Excited & 6.43 & 0.36 & 40 & 51 & 9 & $2.00(128)$ \\
Happy & 10.04 & 0.34 & 38 & 53 & 9 & $3.42(129)$ \\
Irritable & 5.15 & 0.32 & 30 & 55 & 15 & $9.63(120)^{*}$ \\
Nervous & 4.67 & 0.32 & 35 & 46 & 18 & $1.96(115)$ \\
Sad & 3.88 & 0.31 & 43 & 39 & 18 & $0.22(115)$ \\
$\quad$ Upset & 4.36 & 0.31 & 38 & 45 & 17 & $0.69(117)$ \\
Activity & & & & & & \\
$\quad$ Used alcohol & 2.00 & 0.18 & 65 & 15 & 20 & $44.61(113)^{*}$ \\
$\quad$ Exercised & 4.91 & 0.35 & 57 & 26 & 16 & $16.41(118)^{*}$ \\
Socialised & 7.06 & 0.37 & 67 & 28 & 5 & $23.40(134)^{*}$ \\
Had quiz/test & 2.61 & 0.12 & 91 & 6 & 3 & $106.87(137)^{*}$ \\
\hline
\end{tabular}

Note. Data for Experiments 1 and 2 combined. $N=141$. ${ }^{*} p<0.05$.

\section{GENERAL DISCUSSION}

The present study produced two main findings. First, data obtained from the diary-complete group indicated that participants in Experiment 1 accurately estimated the frequency of their emotions and their activities for the 2-week reference period; regardless of item type, signed error was not reliably different from zero, absolute error was small and the rank-order correlation between estimates and actual frequency was high. Second, the types of strategies used to estimate activity frequencies differed from the types used to estimate emotion frequencies. Specifically, when estimating activity frequencies, participants in Experiment 1 and Experiment 2 relied heavily on memory-based strategies (enumeration and directretrieval) and rarely used the self-knowledge strategies (on-average, typical), whereas emotion items elicited both self-knowledge and memory-based responses. Moreover, we found that actual frequency affected strategy selection differently depending on whether people were estimating the frequency of emotions or activities; activity items showed an expected pattern of decline in memory-based strategies as actual frequency increased. In contrast, when the questions concerned emotions, self-knowledge responses were somewhat more common than memory-based responses, regardless of frequency.

These findings have both theoretical and practical importance. From a theoretical perspective the data indicating that self-knowledge and memory-based strategies are both commonly used to estimate emotion frequencies are of particular interest. On the one hand, the frequent use of self-knowledge strategies indicates that abstract emotion knowledge does exist, that this knowledge is fairly well calibrated, and that it can be retrieved and used like other sorts of declarative information. On the other hand, the frequent use of memorybased strategies (i.e. a third of the emotion frequency estimates were generated via memory-based strategies like enumeration) suggests that abstract emotion knowledge might not be exhaustive (i.e. people do not track all possible emotional states), but that they can interrogate autobiographical memory in a way that produces accurate information about recent emotional states. In other words, it appears that the abstract-knowledge approach and the episodic-memory position have merit and that a complete theory of emotion will have to encompass both (Robinson \& Clore, 2002b). 
From a practical perspective, the accuracy of the emotion estimates is an important finding. It is important because it increases our confidence that instruments and questionnaires that use a 2 -week reference period can provide valid information about a respondent's recent emotional experience. On average, participants were only off in their frequency estimates of emotions by 2 days in either direction and they were off by only 1 day when estimating the frequency of activities in a 2-week period. Of course, there is still much work to be done on the issue of accuracy. It will be necessary to determine whether our findings generalise across additional emotion terms and over longer time frames. We will also need to understand whether these findings hold across the life-span and whether (and how) psychiatric problems and substance abuse affect people's ability to generate accurate estimates of emotion frequencies. Admittedly, these are difficult issues. Nonetheless, it does seem that a web-based diary approach of the sort used in Experiment 1 can be extended to assess the accuracy of emotional frequency responses under a wide variety of conditions.

One final point is that it is common practice to take a coarse-grained approach to the assessment of emotion frequency. For example, in Experiment 1, participants indicated whether they had experienced the target emotion at any point during the previous 24 hours and later estimated on how many days they experienced the emotion in the target period (for other examples of coarse-grained assessments, see Cohen et al., 1983; Radloff, 1977; World Health Organization, 1990). This approach does not consider the fact that people experience a wide range of emotions on a daily basis and that these emotional experiences differ in their frequency, duration, intensity and distinctiveness (Kahneman, Krueger, Schkade, Schwarz, \& Stone, 2004; Smith \& Ellsworth, 1985). Admittedly, at this point, we do not understand why people endorse the emotions they do when they are filling out their daily diaries nor can we link the quantitative dimensions of emotional experience to these decisions. Rather we have assumed that people check off items that refer to relatively 'important' emotional experiences and that they will not bother to endorse an item when it refers to experiences that are faint, trivial or ambiguous. Clearly it would be useful to validate these assumptions; this could be done by combining the daily diary method employed in this study with fine-grained emotion monitoring techniques such as the day reconstruction method (e.g. Kahneman et al.) and the experience sampling method (e.g. Larson \& Almeida, 1999). Thus, in addition to determining whether our findings generalise across items, time frames and populations, future research will be needed so that we can better understand how people interpret and encode the subtle shifting stream of emotion that comprises their affective experience and how this interpretation is captured by emotion diaries and reflected in emotional frequency judgements.

\section{ACKNOWLEDGEMENTS}

This research was supported by Social Sciences and Humanities Research Council of Canada grant to Nancy L. Galambos.

\section{REFERENCES}

American Psychiatric Association. (1994). Diagnostic and statistical manual of mental disorders (4th ed.). Washington, DC: Author. 
Barsalou, L. W., \& Ross, B. H. (1986). The role of automatic and strategic processing in the sensitivity of superordinate and property frequency. Journal of Experimental Psychology: Learning, Memory, and Cognition, 12, 116-134.

Blair, E., \& Burton, S. (1987). Cognitive processes used by survey respondents to answer behavioral frequency questions. Journal of Consumer Research, 14, 280-288.

Bolger, N., Davis, A., \& Rafaeli, E. (2003). Diary methods: Capturing life as it is lived. Annual Review of Psychology, 54, 579-616.

Brown, N. R. (1995). Estimation strategies and the judgment of event frequency. Journal of Experimental Psychology: Learning, Memory, and Cognition, 21, 1539-1553.

Brown, N. R. (1997). Context memory and the selection of frequency estimation strategies. Journal of Experimental Psychology: Learning, Memory, and Cognition, 23, 898-914.

Brown, N. R. (2002). Encoding, representing, and estimating event frequencies: A multiple strategy perspective. In P. Sedlmeier, \& T. Betsch (Eds.), Frequency processing and cognition (pp. 37-53). Oxford: Oxford University Press.

Brown, N. R. (2005). On the prevalence of event clusters in autobiographical memory. Social Cognition, 23, 35-69.

Brown, N. R., \& Schopflocher, D. (1998a). Event clusters: An organization of personal events in long-term memory. Psychological Science, 9, 470-475.

Brown, N. R., \& Schopflocher, D. (1998b). Event cueing, event clusters, and the temporal distribution of autobiographical memories. Applied Cognitive Psychology, 12, 305-319.

Brown, N. R., \& Sinclair, R. C. (1999). Estimating number of lifetime sexual partners: Men and women do it differently. Journal of Sex Research, 36, 292-297.

Burton, S., \& Blair, E. (1991). Task conditions, response formulation processes and response accuracy for behavioral frequency questions in surveys. Public Opinion Quarterly, 55, 50-79.

Cohen, S., Kamarck, T., \& Mermelstein, R. (1983). A global measure of perceived stress. Journal of Health and Social Behavior, 24, 385-396.

Conrad, F. G., Brown, N. R., \& Cashman, E. R. (1998). Strategies for estimating behavioral frequency in survey interviews. Memory, 6, 339-366.

Conrad, F., Brown, N. R., \& Dashen, M. (2003). Estimating the frequency of events from unnatural categories. Memory \& Cognition, 31, 552-562.

Cutler, S. E., Larsen, R. J., \& Bunce, S. C. (1996). Repressive coping style and the experience and recall of emotion: A naturalistic study of daily affect. Journal of Personality, 64, 379-405.

Kahneman, D., Krueger, A. B., Schkade, D. A., Schwarz, N., \& Stone, A. A. (2004). A survey method for characterizing daily life experience: The day reconstruction method. Science, 306, 1776-1780.

Klein, S. B., \& Loftus, J. (1993). The mental representation of trait and autobiographical knowledge about the self. In T. K. Srull \& R. S. Wyer, Jr. (Eds.), Advances in social cognitions: The mental representation of trait and autobiographical knowledge about the self (Vol. 5, pp. 1-49). Hillsdale, NJ: Lawrence Erlbaum.

Larson, R. W., \& Almeida, D. M. (1999). Emotional transmission in the daily lives of families: A new paradigm for studying family process. Journal of Marriage and the Family, 61, 5-20.

Levine, L. (1997). Reconstruction memory for emotions. Journal of Experimental Psychology: General, 126, 165-177.

Pandelaere, M., \& Hoorens, V. (2006). The effect of category focus at encoding on category frequency estimation strategies. Memory \& Cognition, 34, 28-40.

Radloff, L. S. (1977). The CES-D scale: A self-report depression scale for research in the general population. Journal of Applied Psychological Measurement, 1, 385-401.

Robinson, M. D., \& Clore, G. L. (2002a). Belief and feeling: Evidence for an accessibility model of emotional self-report. Psychological Bulletin, 128, 934-960.

Robinson, M. D., \& Clore, G. L. (2002b). Episodic and semantic knowledge in emotional self-report: Evidence of two judgment processes. Journal of Personality and Social Psychology, 83, 198215.

Schimmack, U. (2002). Frequency judgments of emotions: The cognitive basis of personality assessment. In P. Sedlmeier \& T. Betsch (Eds.), Etc.: Frequency processing and cognition (pp. 189-204). Oxford, England: Oxford University Press.

Schimmack, U., \& Hartmann, K. (1997). Individual differences in the memory representation of emotional episodes: Exploring the cognitive processes in repression. Journal of Personality and Social Psychology, 73, 1064-1079. 
Smith, C. A., \& Ellsworth, P. C. (1985). Patterns of cognitive appraisal in emotion. Journal of Personality and Social Psychology, 48, 813-838.

Thomas, D. L., \& Diener, E. (1990). Memory accuracy in the recall of emotions. Journal of Personality and Social Psychology, 59, 291-297.

Thompson, C. P. (1982). Memory for unique personal events: The roommate study. Memory \& Cognition, 10, 324-332.

Tiedens, L. Z., \& Linton, S. (2001). Judgment under emotional certainty and uncertainty: The effects of specific emotions on information processing. Journal of Personality and Social Psychology, 81, 973-988.

Watson, D., Clark, L. A., \& Tellegen, A. (1988). Development and validation of brief measures of positive and negative affect: The PANAS scales. Journal of Personality and Social Psychology, 54, 1063-1070.

World Health Organization. (1990). Composite international diagnostic interview (CIDI version 1.0). Geneva, Switzerland: World Health Organization. 